\title{
Gobernanza territorial a través del prisma de los instrumentos, aprendizajes y conflictos
}

\author{
Territorial governance through the prism of tools, learning processes and conflicts
}

\author{
Historial del artículo \\ Recibido: \\ 18 de mayo de 2020 \\ Revisado \\ 19 de junio de 2020 \\ Aceptado: \\ 29 de octubre de 2020
}

\author{
Eduardo Chia ${ }^{\mathrm{a}}$, André Torre ${ }^{\mathrm{b}}$ \\ a Institut National de Recherche pour l'agriculture, l'alimentation et l'environnement INRAE, L'Unité Mixte de Recherche (UMR) \\ Innovation Montpellier, Francia. Correo electrónico: eduardo.chia@inrae.fr \\ b Université Paris-Saclay, Institut National de Recherche pour l'agriculture, l'alimentation et l'environnement INRAE, \\ Agroparistech, Paris, Francia.
}

\section{Palabras clave}

Coordinación territorial, innovación territorial, instrumentos, procesos.

\section{Keywords}

Mechanisms, territorial coordination, territorial innovation, tools.

\section{Resumen}

En este artículo, principalmente a partir de nuestros trabajos realizados en Francia y de nuestra experiencia en varios países de Latinoamérica (Brasil, Uruguay y Chile), proponemos una revisión de las principales dimensiones de la gobernanza territorial, con un énfasis, a través del prisma de los instrumentos, los aprendizajes y los conflictos. Nuestros trabajos sobre la gobernanza territorial son la continuidad de investigaciones, empezadas en los años 80 , sobre el desarrollo territorial y rural, el rol de las explotaciones agrícolas en las dinámicas territoriales, la gestión del medio ambiente en particular del agua, el rol de las proximidades y los conflictos, el rol de los instrumentos, dispositivos y los aprendizajes en las coordinaciones entre los actores. Lo que defendemos aquí, resultado de nuestras investigaciones, es que los territorios son, hoy, entidades - espacios-donde se tomas decisiones estratégicas de mediano y largo plazo de manera participativa dando origen a ciertas formas de gobernanza territorial.

\begin{abstract}
In this article, based mainly on our work in France and our experience in several Latin American countries (Brazil, Uruguay and Chile), we propose a review of the main dimensions of territorial governance with an emphasis, through the prism of tools, learning abilities and conflict behaviors. Our works on territorial governance started in the 1980s. They are devoted to the study of territorial and rural development, the role of farms in territorial dynamics, the management of environmental dimensions, particularly water, the role of proximity and conflicts, and the role played by tools, devices and learning in the coordination between the actors. What we defend here, the result of our research, is that the territories are, today, places where medium and long-term strategic decisions, when they are made in a participatory manner, give rise to various forms of territorial governance.
\end{abstract}




\section{Introducción}

Gobernanza: ¿un nuevo modelo de legitimación de las políticas públicas?

Frente a los fracasos o limitaciones de las autoridades en la implementación de las políticas económicas y sociales, en particular a nivel local, el concepto de gobernanza ha surgido para describir emergentes modos de gobernabilidad; es decir, nuevas prácticas de interacción entre actores públicos, privados y otros actores territoriales que van a generar diferentes capacidades de gobernar. Si bien fue primero una propuesta de algunos organismos internacionales para mejorar la coordinación entre los países y evaluar la participación de las poblaciones en las decisiones públicas, esta fue rápidamente desviada por los actores locales para actuar de manera participativa y los científicos (economistas, cientistas políticos, sociólogos, planificadores y geógrafos) para analizar los nuevos modos de coordinación a nivel territorial. Este concepto se utiliza ahora para analizar o proponer nuevos modos de coordinación, practicados por actores públicos y privados para asegurar los procesos de desarrollo, incluyendo las dinámicas de desarrollo territorial.

Es la gobernanza un concepto que se ha popularizado e invadido nuestro cotidiano, puede parecer, a primera vista, fuerte y reservado solo para acciones ubicadas a alto nivel de jerarquía de Estados y naciones. Y como muestran Glückler, Rehner \& Handke (2019) el concepto de gobernanza es polisémico, lo que es a la vez una ventaja y un inconveniente, ya que permite ser utilizado para analizar situaciones tanto a nivel local como nacional o internacional. Como lo señalan Figueroa-Sterquel \& Chia (2016): "La gobernanza no es solo un fenómeno de moda, sino que también es un desafío político y científico" (p. 2). Sin embargo, se trata simplemente de tomar decisiones, arbitrar oposiciones y conflictos, gestionar modos y procesos de producción, crear instrumentos y mecanismos para gobernar y contribuir a la regulación de las actividades económicas y sociales (cuando sea posible). Una exigencia que se puede encontrar en diferentes niveles de la construcción política, desde la Unión Europea hasta la comuna rural, pasando por una gran región, una comunidad local o un territorio. La idea del gobierno ha dominado durante mucho tiempo los asuntos públicos, con el principio de una jerarquía descendente y restrictiva, apoyándose en las leyes y las políticas públicas. Luego, poco a poco, fue surgiendo el concepto de gobernanza, a veces polisémico e impreciso, pero vinculado a formas de poder más flexibles, basadas en la coordinación de actores, grupos sociales e instituciones para lograr objetivos comunes (Le Galès, 2014). Esta evolución se inscribe en un movimiento de contestación de las políticas públicas y las prácticas de gobierno por parte de los Estados (crisis de legitimidad del Estado), pero también en un contexto de innovaciones institucionales vinculadas a los procesos de descentralización y contractualización entre el Estado y las regiones, las colectividades territoriales.

La aparición del principio de gobernanza responde a los límites de las políticas públicas (nacionales y regionales) cuestionadas principalmente por los ciudadanos que desean participar en la elaboración de estas políticas públicas, para que respondan mejor a sus expectativas. Pero también se hace eco del movimiento de diferenciación de los diferentes componentes de la sociedad-stakeholders-(Le Galès, 1995), de la multiplicación de los actores (Pasquier, Simoulin \& Weisbtein, 2007) y de las demandas de democracia, más allá de la mera representación electoral. Deseosos de participar más en los asuntos públicos, los actores de la sociedad se organizan, para demostrar sus capacidades de aprender e innovar, movilizando en particular la experiencia e influir así en la fábrica de las políticas públicas, como en el caso de los foros híbridos (Callon, Lascoumes \& Barthe, 2001).

La gobernanza posee un lado normativo, con sus preceptos y métodos: la buena gobernanza, defendida por el Banco Mundial o el Fondo Monetario Internacional (FMI), por ejemplo, proponen recetas supuestamente para garantizar la competitividad de los países o regiones. Pero el concepto/ noción hace también referencia a la experimentación de nuevas formas de acción pública y de participación de los actores en la toma de decisiones, en ruptura con el enfoque de gobierno clásico, cuya organización piramidal o jerárquica es cuestionada a favor de formas más flexibles y próximas a las personas y organizaciones. Las redes de actores económicos y sociales, consideradas durante mucho tiempo como profanas, receptoras de decisiones públicas, ven hoy su voluntad y sus capacidades de expertise e innovación tomadas en cuenta en la fábrica de políticas y acciones públicas (Kooiman, 2000). Se trata de la integración de los nuevos socios público-privados en la definición de los objetivos de desarrollo (Wettenhal, 2003), de la participación de diversas organizaciones (sociales, empresas, organizaciones no gubernamentales -ONG-, etc.) en la elaboración de leyes, normas y reglamentos (Pierre, 2000), o de mecanismos que faciliten la participación de partes interesadas o stakeholders, cada vez mejor organizadas e informadas, en los procesos de toma de decisiones. Glückler (2019) propone la idea de una gobernanza lateral de redes para entender "las redes 
organizadas, en el que las empresas ponen en común sus recursos y unen sus intereses en la búsqueda de objetivos comunes" (p. 93). Esta situación se acerca mucho a la que se produjo en Chile con la política de Clúster.

Por lo tanto, la cuestión de la gobernanza se ha convertido en una de las principales preocupaciones de los dirigentes políticos. El hecho de que la gobernanza, sea global, latinoamericana, urbana, medioambiental, rural... se trata de resolver, analizar cuestiones de interacción, acción colectiva y participación, interés general y consulta, y movilizar los diferentes niveles territoriales (Hooghe \& Marks, 2001). La gobernanza multinivel (Bache \& Flinders, 2004), que se refiere a la conjunción de reglas legales (leyes y reglamentos) del Estado, las naciones y las regiones. En el caso de Europa, se debe considerar además el hecho de que las decisiones nacionales no dependen solo de las directivas y prescripciones de las autoridades nacionales, sino también de Bruselas.

Este es también el caso de los territorios, ahora considerados como lugares donde los actores se coordinan, toman decisiones y elaboran proyectos, y por lo tanto aparecen como verdaderas organizaciones o laboratorios de la toma de decisiones. Los actores presentes en los territorios participan en su gobernanza, desarrollando nuevas capacidades para actuar juntos, que nacen y se basan por un lado en procesos de aprendizaje, y por otro, en herramientas, instrumentos y dispositivos. Los conflictos en los territorios provocan otras situaciones creativas, lo que obliga a los actores a buscar nuevas formas de trabajar y decidir juntos, a crear nuevas herramientas, instrumentos y mecanismos de gobernanza.

Dicho de otra manera, hoy, los territorios, al igual que las empresas o las instituciones, pueden ser consideradas como organizaciones (lugares, espacios) donde los actores se coordinan, toman decisiones, elaboran proyectos e innovan. El aprendizaje territorial caracteriza así las nuevas capacidades cognitivas y las nuevas prácticas que los actores adquieren y que les permiten trabajar juntos. Los aprendizajes permiten, a los actores, crear un lenguaje común, definir proyectos compartidos, construir e implementar reglas y procedimientos operativos para evaluar acciones y compartir experiencias. Las herramientas, instrumentos y dispositivos estructuran el comportamiento de los actores y en algunos casos pueden vivir sus propias vidas y escapar a su rol de actores.
Los conflictos son situaciones creativas en términos de gobernanza territorial y obliga a los actores a buscar, inventar y apropiarse de nuevas formas de trabajo: crear un lenguaje, instrumentos, dispositivos como también proyectos comunes y decidir juntos. En algunos casos los actores se apropiarán de las herramientas, instrumentos y mecanismos propuestos por las autoridades, pero a veces se opondrán y protestarán contra las decisiones de las autoridades o de las grandes organizaciones, lo que da lugar a nuevas innovaciones técnicas, y organizacionales, sociales o institucionales. Lo anterior se puede calificar de espiral virtuosa de la innovación.

En este artículo examinamos, a partir de trabajos previos (revisión bibliografía) sobre la gobernanza, los aprendizajes y los conflictos, y nuestros propios trabajos, realizados principalmente en Francia, y los cuales utilizamos aquí, pero también en otros países (Brasil y Uruguay) y en particular en Chile ${ }^{1}$. Los procesos de gobernanza territorial a través del prisma de los dispositivos, aprendizajes y conflictos que la construyen y modelan. Mostramos que es posible y deseable analizar la gobernanza a través de un prisma distinto al de la participación. En primer lugar, damos una definición de la gobernanza territorial, sus principales mecanismos y sus actores. Después, mostramos la importancia de los instrumentos en los sistemas de gobernanza y aprendizaje territorial, antes de abordar la dimensión raramente explorada del papel que desempeñan las relaciones conflictuales.

\section{Definición y utilidad de la gobernanza territorial}

El desarrollo territorial debe poner atención a las estructuras y los métodos de intervención del Estado, como así también a la voluntad de la población. Esta idea, que ahora tiende a arraigarse, ha tardado en afirmarse, esencialmente por dos razones. La primera, corresponde a una visión del desarrollo en la cual había un interés por las estructuras productivas o la innovación, con un interés marcado por la competitividad de los territorios, horizonte ineludible (prioritario) de las políticas públicas locales o desconcentradas. La segunda, tiene sus raíces en los enfoques del desarrollo ascendentes, a menudo basados en el mito de la organización espontánea o natural de los actores locales, garantizando sus derechos de expresión o de acción al margen de las problemáticas organizacionales o de estructuración de la opinión.

\footnotetext{
${ }^{1}$ Ver el número especial (53) sobre la Gobernanza territorial, los aprendizajes y los conflictos, de la Revista de geografía de la Pontifica Universidad Católica de Valparaíso, editado en 2016 http://www.revistageografica.cl/index.php/revgeo/article/
} 


\section{Los orígenes de la gobernanza territorial en las ciencias sociales}

El primer cuestionamiento surgió de las reflexiones sobre la participación de accionarios en las decisiones y orientación de las empresas (Pérez, 2009), en particular de debates sobre las modalidades de organización interna de las empresas y las formas más ventajosas de externalización de actividades (Ménard, 1997). Pero estas preocupaciones se extendieron rápidamente al nivel territorial, en particular sobre la gestión de subcontratistas y proveedores en un mismo ecosistema, y luego sobre las modalidades de gobernanza de los sistemas locales. El éxito de algunos polos tecnológicos o parques (clúster) empresariales de alta tecnología (Silicon Valley) contribuyeron al éxito de la problemática de gobernanza. Efectivamente, su éxito no solo se debe a la competitividad de las empresas o a la calidad de sus redes, sino que también depende de la capacidad de las instancias (formales e informales) locales para gestionar sistemas socioeconómicos complejos y para hacer cumplir las normas aceptadas por todos los participantes.

La mirada espacial de los procesos de gobernabilidad se ha desplazado gradualmente de las regiones a los territorios, con la integración de los actores y sus problemas locales en la definición de los objetivos y la implementación de las decisiones públicas (Subra, 2016). El interés por las cuestiones locales corresponde a la evolución de las políticas -de macroequilibrios hasta el principio de subsidiariedad-y luego a la importancia de la intervención del Estado (Commailles, 2014). Los distintos modelos de relaciones con el poder central se han multiplicado, con sus dimensiones institucional, territorial, de identificación y política (Pasquier, 2012), integrando los niveles económicos y sociales, así como las funciones régaliennes (en francés) (Lascoumes \& Le Galès, 2007). Por último, la tensión entre territorios y el papel tradicional de un Estado que gobierna a distancia, a través de mecanismos públicos y diversos instrumentos (Epstein, 2005), ha acelerado la insatisfacción de las formas tradicionales de gobierno.

\section{La declinación territorial de la gobernanza: la gobernanza territorial}

La gobernanza se impuso poco a poco en el paisaje político y científico, como lo explica Calame (2015, citado por Figueroa-Sterquel \& Chia, 2016), a propósito de la gobernanza territorial:

Hace quince años en el mundo intelectual francés, el término era "tabú" considerado como el caballo de Troya del neoliberalismo y de las instituciones internacionales como el Banco Mundial, deseosa, se decía, de hacer desaparecer el concepto mismo de política en beneficio de un enfoque gerencial, si es posible dominado por las empresas privadas. En cuanto al territorio, concepto relativamente vago ¿No era el medio, en ese caso, también, de escamotear a las instituciones políticas la gestión del espacio? (p. 59)

El pasaje a la gobernanza territorial se hizo entonces evidente (Bertrand \& Moquay, 2004; Chia, Torre \& Rey-Valette, 2008; Leloup, Moyart \& Pecqueur, 2005), y podemos definirla como un proceso de coordinación entre actores de distinta naturaleza (productivos, asociativos, individuos, autoridades públicas o autoridades locales), con recursos asimétricos, reunidos en torno a cuestiones territorializadas y contribuyendo con la ayuda de herramientas y estructuras adecuadas al desarrollo, a veces concertado, pero otras a veces conflictivo, a proyectos comunes para el desarrollo de territorios (Chia, Vitry \& Negrete, 2016; Torre \& Traversac, 2011). Esta evolución marca la transformación de la investigación en torno a los territorios, considerados como lugares de proyecto colectivo y de articulación de relaciones globales-locales (Gilly \& Torre, 2000; Simard \& Chiasson, 2008).

La visión pragmática así desarrollada actúa como punto de inflexión del desarrollo territorial, sin olvidar la articulación local-global, y se preocupa de la construcción de herramientas y representaciones compartidas (Beuret, 2010), así como la de territorios como espacios de proyectos conjuntos o comunes (Rey-Valette et al., 2014). Esta visión responde a ciertos objetivos sencillos:

- contribuir a la elaboración o favorecer la implementación de proyectos de desarrollo e innovaciones;

- facilitar la coordinación entre actores heterogéneos dentro de los territorios;

- impedir que determinados agentes abandonen el territorio (proceso de desertificación o abandono);

- evitar los enfrentamientos bloqueadores;

- decidir de las vías de desarrollo,

- entre otros.

Diversos estudios concluyen a la necesidad de territorializar los objetivos y concuerdan con la inadecuación de los territorios administrativos para promover los cambios y las innovaciones. Por otra parte, estos estudios muestran la ruptura con los enfoques sobre los mecanismos de gobierno a través de la administración y las políticas herméticas y, por otra, la irrupción de cuestiones de democracia de proximidad en los procedimientos de gestión de las 
personas y las organizaciones, así como en la gestión de los territorios. Ellos comparten también el hecho que la evaluación expost de los mecanismos o políticas públicas y la investigación sobre la gobernabilidad haciéndose, debería apuntar a entender los modos de construcción de una cierta representación o proyecto común (Beuret, 2010; Rey Valette et al., 2014) como así también la lógica de fortalecimiento de los procesos democráticos y de transición a una democracia deliberativa, incluso cooperativa (Beuret \& Cadoret, 2010).

\section{Actores y mecanismos de la gobernanza territorial}

Para analizar la gobernanza de los territorios en su complejidad, hay que tener en cuenta en primer lugar los componentes de la acción pública que contribuyen a la toma de decisiones de las autoridades locales o extralocales. Estos son en particular:

- $\quad$ leyes, promulgadas a nivel nacional, que se aplican a territorios específicos, así como a todos los territorios institucionales que representan a una nación;

- reglamentos, ya sean nacionales, de seguridad, sociales, de etiquetado o reglamentos y directivas;

- $\quad$ las políticas públicas, nacionales o descentralizadas a nivel de las grandes regiones francesas: políticas económicas, en términos de desarrollo industrial, de servicios, de agricultura o de energía, por ejemplo; políticas sociales relativas al trabajo, a la vivienda, a la salud, a la educación; o incluso políticas de ordenamiento del territorio, vinculadas a la cuestión de las infraestructuras o a las dimensiones del territorio;

- instrumentos financieros (ayudas, impuestos, contribuciones de los usuarios, etc.) que ayudan a orientar las políticas y los proyectos emprendidos por los actores territoriales.

Sin embargo, la gobernanza territorial es también multinivel, e implica cada vez más intervenciones del Estado central, así como de los niveles supranacionales (en nuestro caso especialmente de la Unión Europea) y subnacionales (principalmente de las regiones). Muy presente en la literatura de ciencias políticas (Bache \& Flinders, 2004), el término surgió a mediados de la década de 1990 para conceptualizar las relaciones, complejas en la Unión Europea, entre los actores del Estado e infranacionales, público y privado, transnacionales y supranacionales, dentro de diversas y múltiples redes de relaciones horizontales y verticales. A nivel territorial, en el caso francés, esto se refiere al hecho de que las decisiones dependen no solo de las órdenes de las autoridades nacionales francesas, sino también de Bruselas, de los servicios descentralizados del Estado y de las autoridades locales.

La gobernanza territorial también se materializa a través de instrumentos concretos de ordenamiento del territorio. Se trata del conjunto de documentos urbanísticos que determinan la manera de habitar y desarrollar los espacios. En el caso francés son los Planes Urbanos Locales (PLU); Planes Regulador Comunal (PRC) en Chile; los consejos de coordinación cuidad puerto en Chile (Zari, 2016); los Planes de Coherencia Territorial (SCoT), los planes de directores regionales y los planes de desarrollo; bien o los diferentes tipos de zonificación resultantes de la acción pública, de la zonificación territorial y de su diversidad que conducen a los miles de pliegos de políticas y apoyos de la gobernanza multinivel (comunidades de municipios y aglomeraciones, parques naturales regionales, proyectos territoriales), o zonificación ambiental (Natura 2000, aves, hábitats, directivas Znieff, corredores ecológicos) ${ }^{2}$, con sus exclusiones y sus complejas áreas de solapamiento y preocupación.

Agreguemos a esto los procesos de coordinación y los proyectos de acción realizados conjuntamente por diferentes categorías de actores territoriales, de carácter privado o semipúblico. Se trata de la cuestión de la democracia participativa y de la participación de numerosos actores locales en un proceso de toma de decisiones y que ya no se deja exclusivamente en manos del Estado. Estos distintos actores actúan de forma concertada, de manera complementaria o en oposición a las autoridades, con la ambición de complementarla, corregirla o inspirarla. Estos actores aparecen y se manifiestan en particular entre dos elecciones, con la idea de que la delegación de poderes otorgada a los representantes electos no les confiere a ellos o al Estado las competencias universales y la capacidad de responder a todas las cuestiones ni a abordar de manera informada nuevos temas.

En el ámbito de la producción, se trata de grupos de actores que tienen proyectos territoriales y estructuran las relaciones locales con diferentes grados de representatividad. Por ejemplo, las cooperativas y grupos de productores en la agricultura y las agroindustrias constituyen los sistemas de grupos de presión, y algunas veces, a pesar de la antigüedad,

\footnotetext{
${ }^{2}$ Se trata de diferentes tipos de zonificación, los cuales fueron iniciados por la Unión Europea, cuyo objetivo es preservar el hábitat natural de las aves, así como la biodiversidad de la fauna y la flora.
} 
están todavía muy arraigados localmente. También las redes de actores para la innovación y la transferencia de tecnología y conocimientos, que intervienen en la producción local o en los sistemas de innovación. Citemos el caso de los polos, de naturalezas y mecanismos de acción variados: como los clústeres (Figueroa-Sterquel, Chia \& Ureta, 2016), distritos industriales o territoriales, polos de competitividad, o las organizaciones de Defensa y Gestión de los productos locales, o los sindicatos de gestión de las cuencas hidrográficas.

Por último, y en un registro más cercano del ordenamiento territorial y del bien común, señalemos el papel creciente de las organizaciones, que marca la irrupción de los ciudadanos en los procesos de toma de decisiones y la participación que pretenden asumir a nivel local, que se trate de llevar a cabo proyectos o que los conteste. Pensamos, en particular, en las organizaciones de protección de la naturaleza, algunas de las cuales extienden su acción al nivel nacional o incluso más allá, y en las organizaciones sociales (centro de madre, de barrio, etc.) que operan a un nivel más microlocal. Durante mucho tiempo, orientados esencialmente a cuestionar las decisiones públicas, se han convertido en stakeholders en el debate público y, en particular, en la elaboración concertada de normas a nivel local, así como en la elaboración y aplicación de protocolos de negociación.

Cabe señalar que los mecanismos de gobernanza territorial que involucran a estos diferentes actores y son el origen de innovaciones e intervenciones de todo tipo, no están totalmente estabilizados y dan lugar a debates y controversias. Sin embargo, existe un consenso bastante amplio sobre la participación de las stakeholders en los debates o en la toma de decisiones. De este modo, la concertación permite construir objetos comunes que pueden facilitar la toma conjunta de decisiones y guiar las decisiones futuras, teniendo en cuenta las limitaciones y la visión de las distintas "partes interesadas" (Touzard, 1972). Al crear las condiciones para la cooperación, por ejemplo, en torno a la concepción colectiva de un proyecto o a la planificación de los usos de un recurso o de un espacio (Beuret \& Cadoret, 2010), contribuye a la construcción de territorios de proyectos comunes, sobre la base de relaciones de coordinación más o menos equilibradas y jerárquicas. Pero tengamos cuidado con la visión idílica de la gobernanza puramente colaborativa y deliberativa. Su funcionamiento, que puede resultar difícil, también se basa en relaciones asimétricas, conflictuales y en oposiciones. Sigue habiendo obstáculos y el éxito del proceso de negociación territorial depende de dos condiciones previas: la aceptación de las reglas del juego por parte de los actores, que pueden retirarse de la situación en lugar de unirse a un proyecto común (Tiebout, 1956) u optar por no expresarse y actuar fuera de los dispositivos de gobernanza, así como la designación de las personas que debaten e implementan los proyectos territoriales y el proceso de desarrollo (Leroux, 2006).

Dispositivos de gobernanza territorial: instrumentos con objetivos estratégicos y políticos

Las nuevas situaciones de gobernanza que están surgiendo en los territorios, en muchos lugares del mundo, se caracterizan por:

(i) la fuerte complejidad de los problemas a abordar y la significativa incertidumbre asociada a los mismos; (ii) el creciente número de actores involucrados y su diversidad;

(iii) la importancia otorgada a las herramientas, instrumentos y dispositivos que requieren que los actores aprendan a apropiárselos, a construir proyectos comunes, a trabajar juntos, a coordinar, y evaluar.

Estas situaciones van a generar aprendizajes de gobernanza que pueden ser considerados como procesos cognitivos y de comportamiento que permiten a los actores del territorio producir una visión compartida del territorio, desarrollar una estrategia y objetivos comunes, y formar y legitimar la acción colectiva (Chia et al., 2016; Vitry \& Chia, 2017). El rol de los instrumentos en la acción colectiva está al centro del paradigma de la gobernanza territorial (Chia, Torre \& Rey-Valette, 2008; Lascoumes \& Simard, 2011; Torre, 2016). Los instrumentos se convierten en auxiliares indispensables (Berry, 1983) para explorar mundos posibles, evaluar las acciones y negociar con el mundo exterior. Estos instrumentos pueden ser fabricados o apropiado por los actores y les permiten, o son utilizados, para coordinarse, lo que es, de vuelta, una nueva fuente de aprendizajes (Argyris \& Schön, 2001).

Entendemos el porqué de la importancia dada a los análisis de las técnicas, a las formas de ordenar los instrumentos (prácticas) en función de los objetivos, del sistema de actores, lo que algunos trabajos presentarán como la tecnología de gobernanza (Chia, Torre \& Rey Valette, 2008; Moisdon, 2005). Al analizar las situaciones de ordenamiento territorial del territorio y de desarrollo territorial hemos observado que un instrumento puede, durante su vida activa, convertirse en un dispositivo. Este es el tema de la siguiente sección.

Instrumentación: hacia una tecnología de gobernanza 
Los trabajos sobre los procesos de gobernanza movilizan de una manera o de otra el trabajo de Foucault sobre los dispositivos (Boussard \& Maugeri, 2003; Chia, Torre \& Rey-Valette, 2008; Hatchuel, Pezet, Starkey \& Lenay, 2005). Recordemos que Foucault (2014), en su definición de dispositivo, habla de un conjunto heterogéneo que abarca discursos, instituciones, desarrollos arquitectónicos, decisiones regulatorias, leyes, medidas administrativas, declaraciones científicas, propuestas filosóficas, morales, filantrópicas, en definitiva, un conjunto heterogéneo tanto de lo que se dice como de lo que no se dice y estos constituyen los elementos del sistema. El dispositivo es entonces la red que se puede establecer entre estos elementos. Un dispositivo sería mucho más completo que un instrumento y el instrumento sería más completo que la herramienta. Veamos cómo estas tres categorías principales de intermediarios de la decisión se articulan: herramientas, instrumentos (o innovaciones de gestión) y mecanismos (tabla 1).

Tabla 1

Definición y características de los intermediarios de la decisión.

\begin{tabular}{|c|c|c|c|}
\hline & Herramienta & Instrumento & Dispositivo \\
\hline Definición & $\begin{array}{l}\text { Extensión de la } \\
\text { mano. }\end{array}$ & $\begin{array}{l}\text { Producto } \\
\text { de una } \\
\text { operación de } \\
\text { pensamiento } \\
\text { superior. }\end{array}$ & $\begin{array}{l}\text { Un instrumento } \\
\text { de alcance } \\
\text { político } \\
\text { relevante para } \\
\text { el desarrollo } \\
\text { de una } \\
\text { estrategia. }\end{array}$ \\
\hline Características & $\begin{array}{l}\text { Equivalente } \\
\text { al sustrato } \\
\text { técnico del } \\
\text { instrumento. }\end{array}$ & $\begin{array}{l}\text { Tiene tres } \\
\text { componentes: } \\
\text { - Sustrato } \\
\text { técnico } \\
\text {-Visión } \\
\text { simplificada de } \\
\text { las relaciones } \\
\text {-Filosofía de } \\
\text { gestión }\end{array}$ & $\begin{array}{l}\text { Un instrumento } \\
\text { transformado } \\
\text { y apropiado } \\
\text { dentro de la } \\
\text { organización: } \\
\text { su visión } \\
\text { simplificada y } \\
\text { su filosofía de } \\
\text { gestión son } \\
\text { transformadas } \\
\text { por los actores. }\end{array}$ \\
\hline
\end{tabular}

Fuente: Vitry \& Chia (2017).

Una herramienta puede ser considerada como la extensión de la mano, un auxiliar útil con un objetivo operativo (Aggeri \& Labatut, 2010.). Su finalidad no se da a priori, sino que emerge o se define en su uso o funcionamiento, por lo tanto, en el uso que los individuos hacen de ella. Las herramientas de gestión incluyen reuniones, matrices de gestión y ordenadores. Para Boussard \& Maugeri (2003) es un montaje (reunión) de elementos materiales, humanos y simbólicos, en interacciones sistémicas entre sí para organizar las actividades humanas, en particular las actividades laborales.

El instrumento, en cambio, es producto de una operación de pensamiento superior (Aggeri \& Labatut, 2010), que es la visión simplificada del funcionamiento de la organización y de las relaciones que permite articular los diferentes objetivos y medios. El instrumento es el que lleva (soporta) el diseño empresarial, permite implementar la filosofía de gestión y la operación del pensamiento superior.

El dispositivo está compuesto por una serie de herramientas que constituyen su sustrato técnico (Hatchuel \& Weil, 1992), tiene una dimensión social y política (Chiapello \& Gilbert, 2013). Algunos investigadores se apoyan en la definición de Foucault de dispositivo para analizar y caracterizar los lugares donde los actores crean significado, lenguaje, proyectos comunes y producen reglas de funcionamiento. Hablan entonces de evaluación, de formación profesional, humanitaria, económica, sanitaria y reglamentaria. Así un dispositivo tiene un objetivo estratégico, implícito o no, y sobre todo apunta a un cambio. Como lo dice Foucault, los dispositivos no son necesariamente objetos o lugares materiales.

Los instrumentos no solo han invadido empresas, organizaciones y territorios (De Gaulejac, 2005), sino que se están desarrollando y reproduciendo muy rápidamente (De Vaujany, 2006). Es cierto que estructuran el comportamiento de los actores. Algunos autores como Halpern, Lascoumes \& Le Galès, (2014) consideran que no hay acción pública autónoma sin sus propios instrumentos. Comprender cómo los actores se apropian o fabrican los instrumentos se convierte en desafío para los decidores públicos y privadas y la investigación. Cuando los actores de un territorio se enfrentan a instrumentos de acción pública (SCOT, PLU, planes de desarrollo, etc.) deben producir nuevos conocimientos y aprendizajes para apropiarse de estos instrumentos o desviarlos.

Relaciones entre actores e instrumentos: la transformación de un instrumento en dispositivo

En una organización o situación territoriales, aquellas que son creadas por una acción particular, como la gestión de un parque regional, algunas acciones de protección del medio ambiente, los instrumentos propuesto por el Estado serán adoptados, apropiados (De Vaujany, 2006), desviados o transformados por los actores en función del sistema de actores, la trayectoria del territorio y los objetivos del momento. Para entender la manera en que estos pueden producir aprendizaje y convertirse 


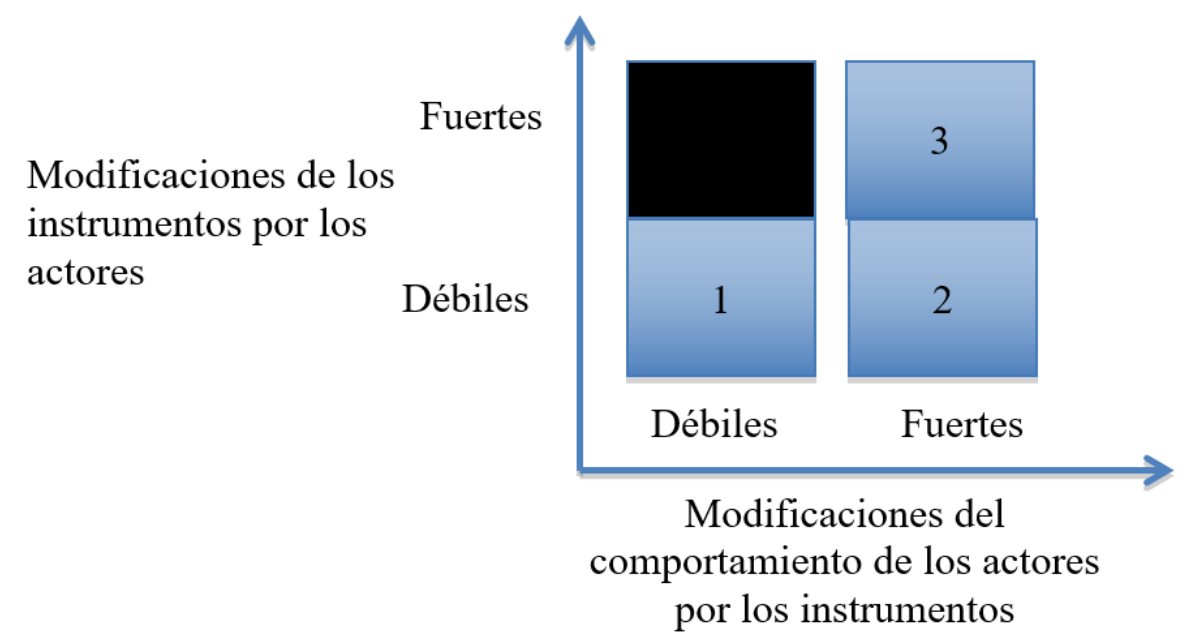

Figura 1. Contextualización de los instrumentos tras la modificación de los instrumentos por los actores y de los actores por los instrumentos. Fuente: Vitry \& Chia (2017).

en dispositivos, proponemos movilizar la noción de contextualización (David, 1998), que toma en cuenta tanto la posibilidad de que los actores modifiquen y se apoderen del instrumento, como la manera en que el instrumento modifica el comportamiento y el conocimiento que los agentes tienen de la situación y de sí mismos: en definitiva, permitirles realizar aprendizajes.

En la figura 1 representamos los cambios fuertes o débiles en el uso de los instrumentos por parte de los actores, así como los cambios en el comportamiento de los actores cuando utilizan el instrumento. Se elimina una de las cuatro situaciones: una fuerte modificación del instrumento por parte de los actores y una débil modificación en el comportamiento de los actores, ya que parece imposible.

En la situación 1, el instrumento es aceptado o utilizado por un pequeño número de personas y tiene poca influencia en la realización de la acción colectiva. En la situación 2, se utiliza el instrumento, sin necesariamente modificarlo. Sin embargo, la utilización de los instrumentos por los actores causa cambios significativos en el comportamiento de estos. Finalmente, en la situación 3, los actores transforman el instrumento y la transformación del instrumento genera cambios en el comportamiento de los actores. El instrumento se transforma en dispositivo, ya que adiciona un rol más (por lo menos) a su rol original. En esta etapa, el instrumento permite a los actores elaborar una estrategia territorial, darle una dimensión política (en el sentido amplio) a la acción. En cada etapa se pueden identificar aprendizajes diferentes y específicos.

\section{El ejemplo de contextualización de un SCOT: un enfoque a través del consenso}

Como acabamos de ver, un instrumento puede convertirse en un dispositivo cuando permite a los actores no solo cambiar la realidad, sino también desarrollar estrategias futuras. Es el caso de la SCOT (Schéma de Cohérence Territoriale), que es un documento de planificación urbana (ordenamiento urbano) que pretende reunir el conjunto de las políticas sectoriales de un territorio, en particular en materia de vivienda, movilidad, desarrollo comercial, medio ambiente y paisaje de la comunidad de municipios. Este instrumento, como los Planes de Desarrollo Comunal (PLADECO) -entre otros- en Chile, es impuesto por las autoridades para armonizar las políticas agrarias y la urbanización. En el caso del SCOT du Lunelois en el sur de Francia (departamento de le Herault) (Chia et al., 2016; Vitry \& Chia, 2017) fue considerado inicialmente como otro instrumento (situación 2, de la figura 1), incluso ignorado por un cierto número de actores comunitarios (situación 1, de la figura 1). Sin embargo, en un momento, tuvieron que organizarse para implementarlo y nacieron una serie de acciones (encuentros, concursos fotográficos, encuestas de opinión, entre otros) que empezaron a producir un lenguaje común y a compartir una cierta representación del territorio. Estas acciones permitieron también enrolar nuevos actores e ir definiendo el objetivo: la filosofía 
de gestión. Frente a las amenazas proclamadas por una gran ciudad vecina, los actores utilizaron el SCOT para elaborar una estrategia (visión política) de futuro común y crearon un mito organizacional (en el sentido de March, 1999): espacio de respiración, el pulmón verde de la región. Esto permitió que la mayoría de los alcaldes de la Comunidad de comunas fuera enrolada (se implicaran) y participaran de manera activa y crítica a la definición del proyecto territorial futuro. Esta transformación fue posible gracias a la contextualización del instrumento: situación 3 de la figura 1.

\section{El aprendizaje territorial: al centro de la gobernanza}

Los actores de la gobernanza necesitan coordinarse en los territorios, no solo para fijar objetivos comunes, sino también para implementar sus decisiones y evaluar sus resultados. Los actores públicos y privados se reúnen allí para tomar decisiones individuales y colectivas que favorecerán a la realización de sus proyectos (económicos, sociales, culturales), los que están basados en parte en herramientas e instrumentos que permiten orientar las acciones, evaluarlas y planificar las actividades. Como proceso adaptativo, la gobernanza pone en juego nuevas prácticas en lo que el conjunto de los actores involucrados debe aprender.

\section{Aprendizaje de la gobernanza organizativa}

Los autores de Management Sciences y constructivistas (Örtenbald, 2001) conciben el aprendizaje como un cambio cognitivo o de comportamiento producido por los individuos y distinguen tres categorías de sujeto aprendices (que aprenden): individual, cuando el individuo aprende solo; colectivo, cuando el grupo produce aprendizaje en conjunto, y organizacional, cuando el aprendizaje ya no está anclado en los individuos que lo produjeron, sino que también en las rutinas de la organización. Argyris \& Schön (2001) propusieron una tipología de bucles (loops): el primer tipo de aprendizaje se refiere a un cambio de prácticas -maneras de hacer-, mientras que el segundo tipo de aprendizaje se refiere a un cambio de metas o valores que dictan u orientan el cambio. Se habla también de un tercer tipo de aprendizaje que sería aprender a aprender.

Tras el trabajo de Berry (1983), Moisdon (2005), Vitry \& Chia (2017) consideramos que los aprendizajes en las organizaciones son activados por los instrumentos al mismo tiempo, porque los individuos toman el instrumento y lo modifican, como porque este instrumento provoca cambios y, por lo tanto, produce aprendizajes. La originalidad de esta proposición es doble. En primer lugar, explora una dimensión reciente del trabajo sobre instrumentación de políticas públicas que enfatiza la importancia de entender el aprendizaje (Halpern, Lascoumes \& Le Galès, 2014). En segundo lugar, participa en la investigación sobre el rol de los instrumentos de gestión en el funcionamiento de las organizaciones, con estudios de caso especialmente interesantes en el campo de la gobernanza territorial, ya que se trata de fenómenos complejos con muchas interacciones verticales y horizontales entre diferentes actores (públicos y privados).

Si muchos autores han establecido la conexión entre el aprendizaje organizacional y la gobernanza, existe, sin embargo, una tendencia a considerar la gobernanza como un vector del aprendizaje y no al revés. En nuestro trabajo sobre los aprendizajes buscamos entender cómo el aprendizaje puede estructurar la acción colectiva y, por lo tanto, la gobernanza territorial. Estos aprendizajes sobre gobernanza son similares a la noción de sensemaking (Weick, Sutcliffe \& Obstfeld, 2005), es decir, un proceso de construcción del sentido común de la acción colectiva que consiste en transformar las circunstancias en situaciones que se formulan explícitamente y sirven como trampolín para la acción.

\section{Los aprendizajes de gobernanza territorial}

Definimos (Vitry \& Chia, 2017) el aprendizaje de gobernanza como todo cambio cognitivo o de comportamiento, individual o colectivo que promueva una evolución positiva o negativa de la gobernanza en un territorio determinado $y$, por lo tanto, de la cooperación y la participación de los interesados en la definición de nuevas opciones relativas a su gestión. Estos pueden incluir procesos que modifiquen modelos mentales compartidos, lo que define una visión común del territorio, construyendo confianza, pero también capacidades organizativas como el encuentro de habilidades de participación, o la capacidad de trabajar juntos.

Estos procesos pueden resumirse en tres categorías y nueve tipos de aprendizaje de gobernanza territorial (tabla 2) (Vitry \& Chia, 2017) que contribuyen respectivamente a:

- Producir una visión compartida. Se trata de dar sentido a lo colectivo y a la acción, de proporcionar un marco de referencia dentro del cual actuar. La visión compartida puede ser la del territorio o la de la acción colectiva.

- Formar, legitimar un colectivo. Se trata de aprender a trabajar juntos, de enrolar a nuevos actores (los interesados) en el (los) proyecto(s) y de construir el sistema de reglas de la gobernanza. 
- $\quad$ Elaborar o revisar una estrategia y objetivos comunes. Estos son el aprendizaje organizacional "clásico" de primer "círculo" (revisar la estrategia, cambiar las prácticas) o en de segundo "ciclo" (negociar un nuevo objetivo).

Tabla 2

Tipología del aprendizaje de la gobernanza territorial.

\begin{tabular}{ll} 
Categorías & \multicolumn{1}{c}{ Tipos } \\
\hline & Crear sentido (sensemaking) \\
\cline { 2 - 2 } $\begin{array}{l}\text { Producir una visión } \\
\text { compartida }\end{array}$ & Coproducción de conocimientos \\
\cline { 2 - 2 } & Dar sentido (sensegiving) \\
\hline $\begin{array}{l}\text { Formar, legitimar un } \\
\text { colectivo }\end{array}$ & Trabajar juntos \\
\cline { 2 - 2 } & Crear interés \\
\cline { 2 - 2 } & $\begin{array}{l}\text { Integración de los principios de la } \\
\text { gobernanza }\end{array}$ \\
\hline $\begin{array}{l}\text { Desarrollar (o revisar) } \\
\text { una estrategia y objetivos } \\
\text { comunes }\end{array}$ & Revisión de las estrategias \\
\cline { 2 - 2 } & Negociar objetivos \\
\hline & \\
\hline
\end{tabular}

Fuente: Vitry \& Chia (2017).

En un trabajo sobre el rol de las cooperativas en la gobernanza territorial en Marruecos, los autores (Vitry, Abdellaoui, Dugue \& Chia, 2015) utilizaron esta clasificación para analizar el rol de las cooperativas y la acción colectiva. Distinguieron:

- Solo las cooperativas creadas en torno a un problema común (organización de la trashumancia y comercialización) y con suficiente tiempo de maduración permitieron a los agricultores aprender a elaborar un sentido común. El problema común (entendido y compartido por todos) como gatillador de la acción colectiva es una condición necesaria para el buen funcionamiento de las cooperativas, pero no parece suficiente para su sostenibilidad.
- La confianza es esencial para aprender mutuamente. Los agricultores y actores institucionales consideran que el éxito de cualquier acción colectiva está condicionado por el grado de confianza existente entre los miembros del grupo cooperativo y hacia un líder que tenga conocimientos técnicos, conozca las leyes, sepa relacionarse además de una cierta moralidad aceptada y anclada en el territorio.

- Aprender a reunirse. Con frecuencia, las reuniones parecen esenciales para el buen funcionamiento de las cooperativas. El presidente de una cooperativa lechera declara que los dirigentes se reúnen mensualmente para discutir de las dificultades encontradas, para lo cual, han creado comités de trabajo encargados no solo de supervisar el buen funcionamiento de los servicios de la cooperativa, sino y sobre todo de detectar los disfuncionamientos.

- Aprender a renovar (cambiar de líder). Es importante establecer un funcionamiento democrático en las estructuras cooperativas y formar nuevos líderes.

Un trabajo similar se realizó en Chile en la región de Valparaíso (Chia et al., 2016) donde se analizaron los aprendizajes producidos por el PREMVAL (Plan Regulador Metropolitano de Valparaíso) y llegamos a las mismas conclusiones, con matices diferentes, pero se creó un mito organizacional:

El alcalde y concejales de Quilpué, de diferentes corrientes político-partidarias, compartieron las representaciones que las organizaciones sociales tenían del área norte de la ciudad, como una zona de conservación ambiental y de parque para la ciudad. Este planteamiento elaboró las bases de un lenguaje común que facilitó encontrar el mito organizacional en la defensa de la conservación ambiental y la conformación de un Parque Metropolitano, en el cual todos los actores de la comuna estaban de acuerdo. Un aspecto importante de mencionar para entender el planteamiento señalado dice relación con el mito identificado, el cual tiene que ver con la puesta en valor de una antigua reivindicación de la población de Quilpué respecto del fundo El Carmen que habría sido donado por su propietario a la comunidad. (Chia et al., 2016, p.65)

Otro ejemplo es el trabajo (Vea \& Chia, 2016) que consistió a analizar en la Región del Libertador Bernardo O’Higgins, la utilización (o el rol) de un SIG en la producción de un lenguaje común entre productores de Quinua, técnicos y decidores locales que facilito la elaboración de un proyecto territorial de implantación del cultivo de Quinua. 


\section{Conflictos}

Podemos observar en todas partes del mundo un aumento de los conflictos en los usos del espacio, lo que se refleja en todo tipo de oposición a las decisiones tomadas por los actores públicos o las grandes organizaciones, oposiciones que se manifiestan particularmente con respecto a las grandes infraestructuras de desarrollo. En países desarrollados como Francia, podemos citar la oposición al proyecto de construcción del aeropuerto de Notre-Dame-des-Landes que duró más de treinta años antes de que el Gobierno lo abandonara, o la represa de Sivens. En otros países, en particular en los países en vías de desarrollo, se puede hablar de conflictos vinculados a grandes infraestructuras como la construcción de grandes represas, por ejemplo, que ha suscitado una oposición muy fuerte en términos de manifestaciones, recursos judiciales, juicios, bloqueo de carreteras o lugares, o incluso, violencia que ha provocado la muerte de algunos opositores en enfrentamientos con la policía (Awakul \& Ogunlana, 2002; Magsi \& Torre, 2013; Swain \& Chee, 2004).

\section{Una lógica de ensayo y error}

Desde el punto de vista normativo, los conflictos por el uso del espacio son designados como obstáculos a la buena gobernanza o incluso antinómica a esta, porque ponen muchos límites a las decisiones de las autoridades o de los operadores privados. Por lo tanto, estas prácticas son opuestas fácilmente a las prácticas o a los mecanismos de consultación o negociación, los que participarían en la buena gobernanza y permitirían llegar a soluciones negociadas, mientras que los conflictos preferirían manifestar una oposición ciega, o comportamientos egoístas como en el caso de Nymby (not in my backyard), en el que la gente solo tiene en cuenta su interés personal, más allá de cualquier consideración del interés colectivo.

Más que las tensiones entre individuos o pequeños grupos, los conflictos de uso plantean la cuestión de la compatibilidad entre los intereses individuales y el interés general. Este último es frecuentemente invocado por las autoridades para defender sus proyectos y afirmar objetivos de desarrollo territorial que supuestamente responden a los intereses del mayor número posible de personas. No solo se puede cuestionar la complementariedad de estos objetivos (en términos de desarrollo económico, de preservación del medio ambiente, de diversidad y de equilibrio entre las actividades de los territorios), sino aún más su compatibilidad con los intereses de los actores de los territorios y la forma en que estos pretenden influir en las orientaciones que vienen desde arriba para hacer prevalecer sus derechos. ¿Son los conflictos de uso un obstáculo para la buena gobernanza? ¿Un simple episodio? Las investigaciones que nosotros hemos llevado a cabo en los últimos diez años nos han permitido de realizar un primer mapa de conflictos y sacar lecciones generales (escalamiento) $^{3}$.

\section{Conflictos y toma de la palabra}

Para situar el lugar que ocupan los conflictos en los procesos de gobernanza territorial, podemos partir de las soluciones presentadas por Hirschman (1970) en caso de desacuerdo entre los actores territoriales, los actores impactados negativamente por las decisiones tomadas por su entorno o que se sienten agredidos por las opciones que no les convienen pueden desarrollar tres comportamientos distintos, correspondientes a diferentes formas de expresar sus diferencias:

- La lealtad (loyalty) consiste en aceptar la decisión tomada y jugar el juego en silencio. Es la aceptación de las decisiones en curso, a través de la participación en las dinámicas y proyectos puestos en marcha, así como a través de la ausencia de oposición pública o la expectativa de una sanción por un paso posterior a través de las urnas.

- La salida (exit) o votar con los pies (Tiebout, 1956) o salir del juego. Es claro que esta solución no siempre es factible, por razones de costos o de precios de los terrenos (por ejemplo, es difícil vender terrenos contaminados a buen precio).

- La toma de la palabra (voice), o voz, consiste en oponerse, legal o ilegalmente, a la decisión tomada y contestarla haciendo uso de la palabra públicamente.

Entre estas soluciones que caracterizan las diferentes vías de gestión de los procesos de gobernanza, en una situación de desacuerdo sobre los procesos de desarrollo territorial (Torre \& Beuret, 2012), es la tercera la que nos interesa particularmente. En efecto, los conflictos se presentan como expresión voice, de la denuncia, cuando toda o parte de la población está insatisfecha con las decisiones

\footnotetext{
${ }^{3}$ Con respecto a una docena de zonas y territorios administrativos en Francia, así como estudios en otros países como Brasil, Chile o Pakistán, se basan en el uso de diferentes fuentes, incluyendo el análisis de litigios y la prensa diaria regional, tal como entrevistas con partes interesadas y expertos, y reuniones de seguimiento.
} 
o proyectos y se siente dejada de lado (descuidada, que no se le toma en cuenta) en las negociaciones o no se encuentran representadas en las estructuras de gobernanza.

La palabra voice puede tomar la forma de una expresión individual, y más bien reservada para pequeños conflictos, o colectivos, con una ampliación del espacio de preocupación y movilización frente a proyectos de gran envergadura o que impliquen una fuerte reconfiguración de las modalidades de gobernanza local (Dowding, John, Mergoupis \& Van Vugt, 2000). En este caso, la oposición se dirige a menudo hacia las autoridades, con el objetivo de cuestionar sus decisiones e influir en el proceso de gobernanza (Martinais, 2015). El objetivo es conseguir que la parte contraria abandone los proyectos previstos o en curso, o influir en la decisión e incorporar algunos de los argumentos y expectativas de los oponentes, o modificar la composición de las partes implicadas en la decisión e incluir a los agentes que se oponen a ella. El impacto, que puede ser considerable, está relacionado con frecuencia con la escala del conflicto y la movilización de la población. En todos los casos, los conflictos ayudan a mantener las interacciones entre los grupos y las partes interesadas, incluso durante las fases más tensas de la oposición, y evitan la ruptura del diálogo.

\section{Conflicto e innovación: una doble relación}

Los conflictos están fuertemente encadenados a los procesos de innovación, al menos si mantenemos una aceptación de la innovación que no se limite únicamente a la tecnología y que integre dimensiones organizacionales, sociales e institucionales (Klein, Laville \& Moulaert, 2014), dándole su función de novedad económica o social. Esta asociación es doble:

- $\quad$ por un lado, los conflictos constituyen buenos indicadores de la innovación, o más bien de resistencia a la innovación y al cambio;

- por otra parte, los períodos de conflictuales corresponden a fases de cambio, durante las cuales se desarrollan nuevas innovaciones y se alteran los equilibrios del pasado.

Los proyectos territoriales, o proyectos en los territorios, traen novedades, innovaciones sociales, técnicas y económicas. Puede tratarse de un nuevo trazado de carretera, una fábrica, la construcción de un parque de ocio, la construcción de oficinas o viviendas, o la construcción de edificios o espacios naturales como parte de un plan de desarrollo local. Estas novedades o cambios pueden provocar resistencia, cristalizar oposiciones más o menos justificadas, o incluso dar lugar a conflictos en caso de implicación de los oponentes. Los grandes cambios, como la construcción de un gran aeropuerto, una central nuclear, un cambio drástico en el uso del suelo, suelen dar lugar a conflictos largos e importantes, por su extensión espacial y sobre todo social, basada en el reclutamiento de nuevos opositores, a veces alejados del escenario de la confrontación.

Pero el conflicto es también una fuente de innovación. Durante su curso, las interacciones, discusiones y oposiciones, la mezcla que provoca, conducen a cambios importantes. En términos de gobernanza, es el momento de la aparición de nuevos actores o grupos, incluso en mesas de diálogos, la reintegración de partes interesadas olvidadas o agraviadas en una fase anterior del desarrollo del proyecto, así como el establecimiento de nuevas jerarquías que reviertan o cambien el orden anterior. Desde el punto de vista técnico, el debate y los intercambios dan lugar a nuevas soluciones innovadoras: nuevos trazados de carreteras, nuevas técnicas de producción de gas a partir de residuos vegetales (metanización o digestión anaeróbica), nuevos métodos de gestión del agua y cambios de uso del suelo.

De esta manera, las fases de conflicto están marcadas por recomposiciones sociales o de grupos de interés, así como por cambios de naturaleza técnica o legal: se pueden probar nuevas alianzas, defender posiciones y confrontar puntos de vista, y se puede rectificar la lista de actores y/o sus representantes en la mesa de negociación. A veces, también, oponerse a la posible adquisición o control de un grupo o individuo sobre el proceso actual, o despedir a un grupo dominante al principio del proceso a favor de grupos emergentes o de la reconstrucción de alianzas. Después del conflicto, quedan nuevos acuerdos a nivel local, nuevos modos de gobernanza, nuevas configuraciones de las mesas de dialogo, así como técnicas (cambios en el trazado de carreteras, desarrollos paisajísticos o técnicos, modificaciones en los planes de uso del suelo, etc.) que resultan de negociaciones previas.

\section{Los conflictos y su impacto en la gobernanza y el desarrollo territorial}

En los países desarrollados, los conflictos de uso rara vez son destructivos o ultraviolentos. Por el contrario, generalmente reflejan oposiciones entre personas que comparten un objetivo o proyecto de desarrollo común, que aceptan o desean vivir juntas dentro de un mismo territorio, pero que no están de acuerdo en cuanto a los medios y técnicas para lograrlo. Motivados por una compleja serie de causas sociales, económicas y técnicas, que van desde la 
divergencia sobre el uso del espacio hasta los procesos de exclusión espacial, pasando por los debates sobre normas u opciones tecnológicas, demuestran la posibilidad de un intercambio democrático mínimo y de un debate abierto sobre los propios medios o fines (sostenibles o industriales, por ejemplo) del desarrollo común. Desde este punto de vista, no es sorprendente que el desafío a los planes de uso del suelo o a los permisos de construcción sea tan importante, porque da sustancia y resultados de opciones divergentes de uso del suelo y desarrollo.

Los conflictos reflejan reacciones y manifestaciones de naturaleza diferente que dan nacimiento a innovaciones, a discusiones sobre su implementación, sobre el proceso de aceptabilidad o su posible rechazo después de la fase de conflicto. Estos son un buen medio para clasificar y seleccionar las buenas innovaciones territoriales, a través de un proceso de prueba y error, y así probar soluciones y rechazarlas si una gran parte de la población las considera inadecuadas. En cierto modo, las situaciones conflictuales son laboratorios de decisión y aceptabilidad (Pelletier, 2014). Cuando se lanzan nuevos proyectos permiten que se prueben:

- En algunos casos, impedirán la realización de estos, si resultan ser demasiado desfasados con respecto a las expectativas de las poblaciones.

- En otros casos, provocarán cambios y modificaciones para que los proyectos sean aceptables y, por lo tanto, puedan llevarse a cabo, aunque sea de forma modificada.

En términos generales, la oposición fundamental se refiere a la pregunta: ¿qué espacios y por lo tanto qué territorios, queremos para mañana? Así, los conflictos no son fases de ruptura con el proceso de gobernanza, sino parte integrante de esta con sus etapas de oposición, que se entrelazan y complementan momentos más consensuados y cooperativos. Como tales, participan en la gobernanza de los territorios, y no pueden concebirse fundamentalmente sin referencia a las fases y estructuras de negociación o consulta, sino más bien en asociación y como complemento de estas últimas (Bobbio \& Melé, 2015; Glazer \& Konrad, 2005, 2007). Las etapas de desarrollo marcan períodos de fuerte progreso e innovación y, por lo tanto, dan lugar a importantes oposiciones, como las fases de bloqueo y desaceleración que pueden conducir tanto a la atonía como a nuevos impulsos.

\section{Conclusión}

El objetivo de este artículo era contribuir a definir mejor el alcance analítico de la gobernanza territorial desde el prisma de los instrumentos, de los aprendizajes y de los conflictos. Como hemos demostrado, la gobernanza territorial puede referirse a situaciones muy diferentes que tienen en común la participación de los actores en el proceso de toma de decisiones a nivel territorial. Pero esta participación puede tomar varias formas. Puede encarnarse en situaciones de cooperación, con el objetivo de integrar y hacer que los actores actúen juntos movilizando dispositivos complejos, herramientas o instrumentos de distinta naturaleza. Pero, por ejemplo, también puede encarnarse en situaciones de conflicto donde la participación de los actores se traduce en una oposición a las decisiones de las autoridades o de las grandes estructuras privadas; durante las cuales se inventan otros modos y forma de gobernanza, o incluso nuevos instrumentos y dispositivos. La participación que sea en situaciones de cooperación o de conflicto genera aprendizajes individuales y colectivos que permiten a los actores no solamente a resolver problemas a corto plazo, sino que también aprender a construir futuros posibles y sobre todo lenguajes y proyectos comunes.

La gobernanza es un fenómeno sociotécnico complejo y situado (contextualizado) y por lo tanto no se trata de producir una norma. A la diferencia de los enfoques que tratan (o tienen por objetivo imponer) una buena gobernanza, la gobernanza debe ser más como un programa de investigación; sobre todo la manera en que los actores construyen el vivir juntos y, por lo tanto, la manera en la construyen sus territorios día tras día según un proceso complejo y diversificado pero que se renueva sin cesar. Por esto, debemos seguir explorando nuevas situaciones de gobernanza territorial para mejorar nuestra capacidad analítica. Para entender la complejidad de la gobernanza, como lo muestran los numerosos artículos ya publicados y los de este número especial en particular, debemos analizarla desde varias aristas o prisma.

Se debería continuar con la investigación de los cambios en los territorios para mejorar, por una parte, los conocimientos sobre los mecanismos (coordinación, participación, etc.) de gobernanza territorial y, por otra parte, el rol de los aprendizajes en las innovaciones territoriales. Para esto, nos parece que se pueden estudiar:

i) las situaciones conflictuales, ya que en estas situaciones los actores se ven obligados a generan acuerdos y abandonar algunos objetivos, lo que produce innovaciones territoriales, sociotécnicas y organizacionales. ¿Cómo se generan? ¿Son estables en el tiempo (sustentables)? ¿Influencian las políticas públicas?; 
ii) las situaciones de aprendizajes provocadas por los instrumentos de acción pública. ¿Cómo los actores aceptan o reciben los instrumentos? ¿Cómo estos instrumentos permiten o no a los actores de elaborar proyectos y/o lenguajes comunes? ¿Cómo los actores transforman, desvían o adoptan los instrumentos?

\section{Referencias}

Aggeri F. \& Labatut J. (2010). La gestion au prisme de ses instruments. Une analyse généalogique des approches théoriques fondées sur les instruments de gestion. Finance Contrôle Stratégie, 13(3), 5-37. Recuperado de https:// www.researchgate.net/publication/254405408 La_gestion_au_prisme_de_ses_instruments_Une analyse_genealogique_des_approches_theoriques_ fondees_sur_les_instruments_de_gestion

Argyris C. \& Schön D.A. (2002). Apprentissage organisationnel : théorie, méthode, pratique. Paris : De Boeck Université.

Awakul, P. \& Ogunlana, S. O. (2002). The effect of attitudinal differences on interface conflict on large construction projects: The case of the Pak Mun Dam project. Environmental Impact Assessment Review, 22(4), 311-335. https://doi.org/10.1016/ S0195-9255(02)00007-0

Bache, I. \& Flinders, M. (2004). Multi-level Governance. New York: Oxford University Press. https://doi.org/1 0.1093/0199259259.001.0001

Berry, M. (1983). Une technologie invisible - L'impact des instruments de gestion sur l'évolution des systèmes humains. Recuperado de https://hal. archives-ouvertes.fr/hal-00263141/document

Beuret, J.E. (2010). De la négociation conflictuelle à la négociation concertative : un point de passage transactionnel. Négociations, 1(13), 43-60. https:// doi.org/10.3917/neg.013.0043

Beuret, J.E. \& Cadoret, A. (2010). Gérer ensemble les territoires : vers une démocratie coopérative. Paris : Editions Charles Leopold Mayer.

Bertrand, N. \& Moquay, P. (2004). La gouvernance locale, retour à la Proximité. Economie Rurale, 280, 77-95. https://doi.org/10.3406/ecoru.2004.5474
Bobbio L., Melé P. (2015). Introduction - Les relations paradoxales entre conflit et participation. Participations, 3(13), 7 - 33. http://dx.doi. org/10.3917/parti.013.0007

Boussard V. \& Maugeri, S. (dir.). (2003). Du politique dans les organisations, sociologies des dispositifs de gestion. Paris : L'Harmattan.

Calame, P. (2015). La gouvernance territoriale, clé de la transition vers des sociétés durables. L'Économie politique, 4(68), 59-70. https://doi.org/10.3917/ leco.068.0059

Callon, M., Lascoumes, P. \& Barthe, Y. (2001). Agir dans un monde incertain. Essai sur la démocratie technique. Paris : Seuil.

Chia, E., Torre, A. \& Rey-Valette, H. (2008). Vers une «technologie» de la gouvernance territoriale! Plaidoyer pour un programme de recherche sur les instruments et dispositifs de la gouvernance des territoires. Norois, 209, 167-177. https://doi. org/10.4000/norois.2603

Chia, E., Vitry, Ch. \& Negrete, J. (2016). La producción de sentido y contextualización de los instrumentos de planificación territorial. Los casos de Lunelois (Francia) y Quilpué (Chile). Revista Geográfica de Valparaíso, 53, 41-71. Recuperado de http://www. revistageografica.cl/index.php/revgeo/article/view/4

Chia, E., Rey-Valette, H, Michel, L., Soulard, Ch., Nougarèdes, B., Mathé, S., Barbe E., Maurel, P., Jarrige, F. \& Guiheneuf, P.Y. (2016). Proposición metodológica para el análisis de la gobernanza territorial a partir de una experiencia francesa. Revista Geográfica de Valparaíso, 53, 23-46. Recuperado de http://www.revistageografica.cl/index.php/revgeo/ article/view/4

Chiapello, E., \& Gilbert, P. (2013). Sociologie des outils de gestion. Introduction à l'analyse sociale de l'instrumentation de gestion. Paris : La Découverte.

Commailles, J. (2014). Sociologie de l'action publique. En Boussaguet L., Jacquot S., Ravinet P. (eds), Dictionnaire des Politiques Publiques. Paris : Presses de SciencesPo. 
David, A. (1998). Outils de gestion et dynamique du changement. Revue Française de Gestion, 4(120), 44-59. Recuperado de https://basepub.dauphine.fr/ handle/123456789/12567

De Gaulejac, V. (2005). La Société malade de la gestion. Idéologie gestionnaire, pouvoir managérial et harcèlement social. Editions le Seuil.

De Vaujany, F. (2006). Pour une théorie de l'appropriation des outils de gestión: vers un dépassement de l'opposition conception-usage. Management \& Avenir, 9, 109-126. https://doi.org/10.3917/ mav.009.0109

Dowding K., John P., Mergoupis T. \& Van Vugt M. (2000). Exit, voice, and loyalty: Analytic and empirical developments. European Journal of Political Research, 37(4), 469-495. https://doi. org/10.1111/1475-6765.00522

Epstein, R. (2005). Gouverner à distance. Quand l'Etat se retire des territoires. Esprit, 11, 96-111. Recuperado de https://halshs.archives-ouvertes.fr/ halshs-00130034

Figueroa-Sterquel, R. \& Chia E. (2016). Gobernanza territorial, conflictos y aprendizajes. Revista geográfica de Valparaíso, 53,1-6. Recuperado de http://www.revistageografica.cl/index.php/revgeo/ article/view/4

Figueroa-Sterquel, R., Chia, E. \& Ureta, P. H. (2016). Estudio del clúster como un instrumento de gobernanza territorial en Chile: el caso de la Palta en la Región de Valparaíso. Revista geográfica de Valparaiso, 53, 139-159. Recuperado de http://www. revistageografica.cl/index.php/revgeo/article/view/4

Foucault, M. (2014). Seguridad, territorio, población. Fondo de Cultura Económica.

Glazer, A., \& Konrad, K.A. (eds). (2005). Conflict and Governance, Springer Verlag. http://dx.doi. org/10.1007/978-3-662-05121-4

Gilly, J.P. \& Torre, A. (2000). Proximidad y dinámicas territoriales. En F. Boscherini \& L. Poma (eds.), Territorio, conocimiento y competividad de las empresas: el rol de las instituciones en el espacio, pp. 259-294. Buenos Aires: Miño y Dávila Editores.
Glückler, J., Rehner, J. \& Handke, M. (2019). Gobernanza, redes y territorio. Revista de Geografia Norte Grande, 74, 5-20. http://dx.doi.org/10.4067/ S0718-34022019000300005

Glückler,J.(2019). Gobernanza lateral deredes: Legitimidad y delegación relacional de la autoridad decisoria. Revista de Geografia Norte Grande, 74, 93-115. http://dx.doi. org/10.4067/S0718-34022019000300093

Halpern, Ch., Lascoumes, P. \& Le Galès, P. (dir.).(2014). L'instrumentation de l'action publique. Paris : Presses de Sciences Po.

Hatchuel A. \& Weil, B. (1992). L'expert et le système, suivi de quatre histoires de systèmes-experts. Paris : Económica.

Hatchuel, A. Pezet, E., Starkey K. \& Lenay, O. (dir). (2005). Gouvernement, organisation et gestion: héritage de Michel Foucault. Les presses de l’Université de Laval.

Hirschman, A.O. (1970). Exit, Voice and Loyalty Responses to decline in firms, organizations, and states. Cambridge (MA): Harvard University Press.

Hooghe, L. \& Marks G. (2001). Multi-level Governance and European Integration. Lanhamn, MD: Rowman \& Littlefield.

Klein, J.L., Laville, J.L. \& Moulaert, F. (eds.). (2014). L'innovation sociale. Toulouse : Eires Editions.

Kooiman, J. (2000). Societal governance: levels, modes, and orders of social-political interaction. En J. Pierre. (ed.), Debating Governance. Authority, steering and democracy. Oxford: Oxford University Press.

Lascoumes P. \& Simard L. (2011). L'action publique au prisme de ses instruments. Introduction. Revue française de science politique, 61(1), 5-22. http:// dx.doi.org/10.3917/rfsp.611.0005

Lascoumes, P. \& Le Galès P. (2007). Sociologie de l'action publique. Paris : Armand Colin, Collection 128.

Lascoumes, P. \& Le Galès P. (2014). Instrument. En L. Boussaguet, S. Jacquot \& P. Ravinet (dir.), Dictionnaire des politiques publiques (4ème édition), pp. 325-334. Paris : Les presses de Sciences Po. 
Le Gales P. (1995). Du gouvernement des villes à la gouvernance urbaine. Revue française de science politique, 45(1), 57-95. Recuperado de https://www. jstor.org/stable/43119350

Le Galès, P. (2014). Gouvernance. En L. Boussaguet, S. Jacquot S \& P. Ravinet (eds.), Dictionnaire des Politiques Publiques. Paris : Presses de Sciences Po.

Leloup, F., Moyart, L. \& Pecqueur, B. (2005). La gouvernance territoriale comme nouveau mode de coordination territoriale? Géographie, Economie, Société, 4(7), 321-332. http://dx.doi.org/10.3166/ ges.7.321-331

Leroux, I. (2006). Gouvernance territoriale et jeux de négociation. Pour une grille d'analyse fondée sur le paradigme stratégique. Négociations, 6, 83-98. http:// dx.doi.org/10.3917/neg.006.98

Magsi, H. \& Torre, A. (2013). Approaches to Understand Land Use Conflicts in the Developing Countries. The Macrotheme Review, 2(1), 19136. Recuperado de https://www.researchgate.net/ publication/234078875 Approaches to understand land_use_conflicts_in_the_developing_countries

Martinais, E. (2015). Le conflit comme mode de participation. Les habitants contestataires de la politique de prévention des risques industriels. Participations, 3(13), 89-117. Recuperado de https://www.cairn.info/revue-participations-2015-3page-89.htm

March, J.G. (1999). Les mythes du management. Gérer et Comprendre, 5-12. Recuperado de http://annales. org/site/gc/1999/gc09-99/04-12.pdf

Ménard, C. (1997). Le pilotage des formes organisationnelles hybrides. Revue économique, 741-750. http://dx.doi.org/10.2307/3502858

Moisdon, J.Cl. (2005). Comment apprend-on par les outils de gestion? Retour sur une doctrine d'usage». En Philippe Lorino (ed.), Entre connaissance et organisation: l'activité collective, pp. 239-250. Paris : La Découverte.

Örtenblad, A. (2001). On differences between organizational learning and learning organization. Learning Organization, The, 8(3), 125-133.
Pasquier, R. (2012). Le pouvoir régional. Paris : Presses de Sciences Po.

Pasquier, R., Simoulin, V. \& Weibstein J. (2007). La gouvernance territoriale. Pratiques, discours et théories. Paris : LGDJ.

Pelletier, M. (2014). La décision territoriale en conflit: ¿Un outil d'évaluation de la participation citoyenne? Géocarrefour, 89(4), 271-282. http://dx.doi. org/10.4000/geocarrefour.9598

Pérez, R. (2009). La gouvernance d'entreprise. Paris : La découverte; Repères.

Pierre, J. (ed.). (2000). Debating Governance. Authority, Steering and Democracy. Oxford: Oxford University Press.

Rey-Valette, H., Chia, E., Mathe, S., Michel, L., Nougaredes, B., Soulard, Ch. Maurel P., Jarrige F., Barbe E. \& Guiheneuf P-Y. (2014). Comment analyser la gouvernance territoriale? Mise à l'épreuve d'une grille de lecture». Géographie, Economie, Société, 1(16), 65-89. http://dx.doi.org/10.3166/ges.16.65-89

Simard, J.F. \& Chiasson G. (2008). La gouvernance territoriale, un nouveau regard sur le développement. Canadian Journal of Regional Science, 31(3), 455 - 471. Recuperado de http://www.cjrs-rcsr.org/ archives/31-3/Intro-FR-final2.pdf

Subra, Ph. (2016). Géopolitique Locale. Paris : Armand Colin. Recuperado de . http://dx.doi.org/10.3917/ arco.subra.2016.01

Swain, A. \& Chee A.M. (2004). Political Structure and 'Dam' Conflicts: Comparing Cases in Southeast Asia. http://www.worldwatercouncil.org/fileadmin/wwc/ Library/Publications_and_reports/Proceedings Water_Politics/proceedings_waterpol_pp.95-114.pdf

Tiebout, Ch. (1956). A pure theory of local expenditures. Journal of Political Economy, 64(5), 416-424. https:// doi.org/10.1086/257839

Torre, A. \& Beuret, J.E. (2012). Proximités territoriales. Construire la gouvernance des territoires, entre conventions, conflits et concertations. Paris : Economica - Anthropos. 
Torre, A. \& Traversac, J.B. (eds). (2011). Territorial Governance. Local Development, Rural Areas and Agrofood Systems. Heidelberg \& N. York: Springer Verlag. http://dx.doi.org/10.1007/978-3-7908-2422-3

Torre, A. (2016). El rol de la gobernanza territorial y de los conflictos de uso en los procesos de desarrollo de los territorios. Revista Geográfica de Valparaíso, 53, 7-22. Recuperado de http://www.revistageografica.cl/ index.php/revgeo/article/view/4

Touzard, H. (1972). La médiation et la résolution des conflits. Paris : PUF.

Veas, C. \& Chia E. (2016). Aprendizajes de la utilización de los SIG en los procesos de construcción de gobernanza territorial: el caso de la re-introduccion de la producción de quinua en la región del Libertador O’Higgins. Revista Geográfica de Valparaíso, 53, 106-120. Recuperado de http://www.revistageografica.cl/index.php/revgeo/article/view/4

Vitry, Ch., Abdellaoui, H., Dugue, P. \& Chia, E. (2015). Apprendre a cooperer: un defi pour l'adhesion des agriculteurs au plan Maroc vert. New Medit, 2, 13-2.
Recuperado de http://agritrop.cirad.fr/577658/

Vitry, Ch. \& Chia, E. (2017). Stratégies d'opposition des acteurs à l'enrôlement. Management \& Avenir, 97, 149-167. https://doi.org/10.3917/mav.097.0149

Weick, K. E., Sutcliffe K. M. \& Obstfeld, D. (2005). Organizing and the Process of Sensemaking. Organization Science, 16(49), 409-421. https://doi. org/10.1287/orsc. 1050.0133

Wettenhall, R. (2003). The rhetoric and reality of public-private partnerships. Public Organization Review, 3 (1), 77-107. https://doi.org/10.1023/A:1023000128175

Zari, S. (2016). Los consejos de coordinación cuidad puerto: ¿instrumento de gobernanza territorial? Revista Geográfica de Valparaíso, 53, 92-105. Recuperado de http://www.revistageografica.cl/index.php/ revgeo/article/view/4 